\title{
Scientific Methods Must Be Public, and Descriptive Experience Sampling Qualifies $^{1}$
}

\author{
Gualtiero Piccinini
}

$5 / 4 / 2010$

\section{Must Scientific Methods Be Public?}

Hurlburt and Schwitzgebel's groundbreaking book, Describing Inner Experience: Proponent Meets Skeptic, examines a research method called Descriptive Experience Sampling (DES). DES, which was developed by Hurlburt and collaborators, works roughly as follows. An investigator gives a subject a random beeper. During the day, as the subject hears a beep, she writes a description of her conscious experience just before the beep. The next day, the investigator interviews the subject, asks for more details, corrects any apparent mistakes made by the subject, and draws conclusions about the subject's mind. Throughout the book, Schwitzgebel challenges some of Hurlburt's specific conclusions. Yet both agree-as I do-that DES is a worthy method.

Why is DES legitimate? In recent years, there's been a serious debate about the legitimacy of methods - such as DES - that rely on so-called "first-person data". ${ }^{2}$ Privatists maintain that such methods are "first-person" or private, thus different in kind from ordinary scientific methods (Chalmers 2004, Gertler 2009, Goldman 1997, Hatfield 2005, Varela 1996, Varela and Shear 1999). Publicists maintain just the opposite: such methods are "third-person" or public, just like other legitimate scientific methods (Dennett 2007, Haybron 2008, Nahmias 2002, Piccinini 2003a, 2009).

Surprisingly, Hurlburt and Schwitzgebel assert neutrality on and even indifference to whether DES is public or private:

Eric [Schwitzgebel]: ... This debate has captured the attention of consciousness studies researchers and the interested public, because it seems to concern the fundamentally important question of how to study consciousness - what sorts of methods can and cannot, should and should not be implemented. So ... the question seems to arise: Are we using here a "firstperson" or a "third-person" method?

Actually, I don't know. Nor do I care, much. The seeming centrality of this debate to the methodology of consciousness studies is an illusion. One looks in vain for any genuine prescriptive differences, any study or method permitted by Goldman or Chalmers, forbidden by

\footnotetext{
${ }^{1}$ Thanks to Russell Hurlburt, Marcin Miłkowski, and Eric Schwitzgebel, and the audience at the 2010 Pacific APA meeting for helpful discussions on this topic and to Brit Brogaard, Jordan Dodd, and Jim Virtel for comments. ${ }^{2}$ Terminological note: the term 'first-person data' is often used to imply that first-person data are private (and the methods using them are private). Here I use 'first-person data' more neutrally, to pick out the relevant type of data without implying that they are either public or private.
} 
Dennett (as Dennett himself notes). The dispute really concerns only the description of introspective methods. Should we describe the interviews in this book (per Chalmers) as "irreducibly first-personal" because they depend on Melanie' $s^{3}$ attunement to her subjective experience? Or should we describe them (per Dennett) as "third-personal" and "objective" because what we are doing is analyzing spoken utterances, in principle available to all, and hypothesizing about what might lie behind them?

Each way of speaking highlights important aspects of the study of consciousness. But the more important question for consciousness studies - what should be the central methodological question-is when and under what conditions and to what extent people's reports about their experience are trustworthy. That, of course, is the topic of this book.

Russ [Hurlburt]: I could not agree with you more, Eric! Fascination with the Goldman-ChalmersDennett debate has misdirected consciousness researchers away from what you rightly call the central question.

Yet let me also say that I consider DES to be a first-person-plural method: We (Melanie, you, and I) examined Melanie's inner experience and evaluated her/our characterizations thereof. To be sure, only Melanie had access to the experience we sought to examine. However, only I had experience with a method designed to identify specific moments, focus attention on those moments, bracket presuppositions, avoid faux generalizations, and so on; and you brought your own perspective that changed and illuminated things. But my use of the term "first-person" here is not intended to imply a position in the Goldman-Chalmers- Dennett debate. I want only to emphasize the value of the skilled investigator-willing participant alliance (217, emphasis in second and fourth paragraphs added). ${ }^{4}$

I agree with Hurlburt and Schwitzgebel that the central methodological question is "when and under what conditions and to what extent people's reports about their experience are trustworthy" (cf. Piccinini 2003, 143).

I also agree that DES is "a first-person-plural" method, in the sense that it requires an unusual degree of interaction and cooperation between investigators and subjects (cf. Jack and Roepstorff's "second person perspective" in their 2002, Box 2). But as Hurlburt points out (and contra Jack and Roepstorff), this is not a way to take a stance in the debate over publicity vs. privacy of methods, or a third option in addition to publicism and privatism. Whether DES is public or private (in the relevant sense) remains to be determined.

DES is as good a candidate as any for a method that relies on private data. For not only does DES rely on subjects' private access to their experience; in addition, DES subjects operate in uncontrolled natural environments and describe the most idiosyncratic features of their experiences. In these respects, DES contrasts with methods such as those studied by Ericsson and Simon (1993), in which several subjects

\footnotetext{
${ }^{3}$ Melanie is the fictitious name of the subject interviewed by Hurlburt and Schwitzgebel in their book.

${ }^{4}$ Unless otherwise noted, page numbers refer to Hurlburt and Schwitzgebel 2007.
} 
verbalize their mental states while performing the same, highly constrained task under controlled environmental conditions. Because of these features of DES, many DES data cannot be validated by reliability studies and validity-checking tests that require comparing multiple subjects (cf. 33).

Nevertheless, I will argue as follows. First, whether a method is public is important, because non-public methods are scientifically illegitimate. Since Hurlburt and Schwitzgebel care about the science of consciousness (and more generally, of mind), they should care about method publicity. Second, and contrary to what Schwitzgebel suggests, there are genuine prescriptive differences between privatism and publicism. Third, DES is a public method-and it's (implicitly) treated as such by both Hurlburt and Schwitzgebel.

One caveat. Schwitzgebel is right that, at least in recent years, the debate over publicism vs. privatism has been especially heated in the consciousness studies literature. But this is a historical contingency. Whether scientific methods should be public is a question that pertains to all of science. And the legitimacy of methods that rely on subjective reports-and first-person data more generally-pertains not only to the study of consciousness but to the study of the mind in general. ${ }^{5}$

\section{Why Method Publicity Matters}

We can't afford to remain neutral. The requirement that scientific methods be public is a fundamental aspect of science. I will now argue that if we reject this publicity principle, we open the way for pseudoscience and bad science. So we should accept it. But then, if our proposed methods of investigatione.g., DES-were private, we should reject them. Therefore, it's important to establish that our methods are public.

A method is public just in case different investigators can apply the method to answer the same questions and, when they do, they obtain the same data. Otherwise, a method is private. ${ }^{6}$ Now consider what happens if we allow private methods.

The following is a putative method for observing people's auras. Place someone against a white background. Focus your attention on the middle of their forehead (their "Brow Chakra" or "Third Eye") for 30 seconds or more. Concentrate very hard. Then, with your peripheral vision, you will see a

\footnotetext{
${ }^{5}$ In this context, the distinction between mind and consciousness may appear to lack a difference. Someone might argue that if a state is accessible to methods that rely on subjects' reports or other first-person data, it must be a conscious state; therefore, the use of first-person data always belongs in the study of consciousness. But this is a non sequitur. Two points. First, studying mental processes some of whose states are conscious (and hence accessible via first-person data) is not the same as studying "consciousness". Researchers rely on first-person data to study problem solving, schizophrenia, subjective wellbeing, and many other mental phenomena. These studies count as studying consciousness only if the term 'consciousness' is used in such a broad sense as to be virtually synonymous with 'mind'. Second, the concept of consciousness is somewhat nebulous-certainly more nebulous than the concept of mind. There are many things that might be meant by 'consciousness', not all of which are directly related to what can be accessed via first-person data. By contrast, 'mind' is a generic umbrella term that includes the relevant phenomena.

${ }^{6}$ For a defense of this definition of method publicity, and a more detailed defense of method publicity, see Piccinini 2003b.
} 
colored halo surrounding the person. That's the aura. (I didn't make this up-there are people making real money with this.)

Why is the study of auras pseudo-scientific? Surely, not for lack of putative methods to observe them or people who claim to observe them. There are plenty of those. Yet most of those who apply the above method would claim not to see any auras, even though the method does not require any special qualifications or expertise. This is the mark of a private method: different investigators, even though they apply the same method to the same questions, get different results. If private methods were legitimate, we should conclude that the study of auras is part of science. All we need to do is rely on those specially gifted individuals who can see auras. Since private methods are illegitimate, however, we may conclude that this putative method for studying auras is spurious.

With publicity comes independent data validation. If different investigators can generate the same data, they can also look for correlations between those data and other data and phenomena, thereby providing independent evidence that the original data are valid. Conversely, with privacy comes lack of public validation. If many investigators cannot generate the same data, they cannot look for independent evidence that such data are valid. Privatists about first-person data are the first to draw the relevant conclusion: first-person data cannot be validated by public means, precisely because others cannot replicate the data (Chalmers 2004, Gertler 2009, Goldman 1997, Hatfield 2005). The same conclusion holds for the output of other private methods, e.g., data about auras. Since most investigators cannot replicate the data, they cannot establish or investigate any correlation between auras and anything else. The reasonable conclusion is that auras are fictions. By the same token, we should reject any theoretical posit that can only be (putatively) observed via private methods.

First Objection? ${ }^{7}$ : The publicity requirement is too strong. Scientific experiments are not always repeated or even repeatable. Many data are accepted by the scientific community without anyone else reproducing them. In some cases, obtaining the same data may even be impossible. For instance, a lone astronomer may see a series of events in the sky, which events no other instrument or observer records. But why should this be scientifically illegitimate? It should not. By the standard that scientific methods must be public, much of science would be rejected. Therefore, it's the publicity principle that must go.

Reply: The objection misconstrues the publicity principle. The principle is not that all experiments should be repeated and all data reproduced. It's that experiments should be repeatable and data reproducible. Even in the case of lone astronomers and other unique observers of unique events, their data are reproducible in the relevant sense. For nothing prevented other observers from observing the same events, or instruments from recording them. If other observers or instruments had been present, they would have recorded the same events. We believe this because there are enough other skilled observers who share enough knowledge of the relevant methods for us to be confident that, were other skilled observers to have applied the same methods under the same circumstances, they would have

\footnotetext{
${ }^{7}$ This objection was inspired by exchanges with Eric Schwitzgebel and Allan Franklin; an explicit argument along these lines, to the effect that method publicity should be rejected altogether, is in Goldman 1997. My present reply is partially indebted to an exchange with Marcel Weber; see also Piccinini 2003a, b, and 2009.
} 
obtained the same data. Not so in the case of auras. Although some people claim to observe auras, they cannot teach their method to other unbiased parties. Thus, their method is not public.

Second Objection ${ }^{8}$ : Still, first-person data are private, so there must be something wrong with your argument. People's epistemic access to their mental states is more direct and accurate than that of external observers. It's epistemically different in kind. In this sense, mental states are private. Firstperson data are generated by people who rely on their special epistemic access to their mental states. Therefore, first-person data-such as the data DES relies on-are private. In addition, first-person data are too useful to discard; therefore, there is something wrong with the publicity principle.

Reply: There are two claims here, one about directness and one about accuracy. I agree that people have more direct epistemic access to (some of) their mental states than external observers do. It's their mental states-no one else has them! In this sense, mental states are private and people have private access to them. But that's not the issue. The issue is whether first-person data, and methods relying on them, are private. The privacy of first-person data and relative methods doesn't follow from the fact that mental states are private. ${ }^{9}$ Nor does it follow from the claim that people have more accurate access to their mental states. But in any case, people do not have more accurate access to their mental states than external observers, so long as external observers are allowed to ask people what's in their mind. If they are, then external observers have equal or more accurate access to people's mental states. For in addition to relying on people's reports, external observers may (i) lack some of the biases that subjects have towards themselves and (ii) avail themselves of independent evidence that is unavailable to the subjects. Thanks to (i) and (ii), external observers may be able to correct some of the subjects' mistakes in accessing their mental states. Therefore, depending on the situation, external observers may have more accurate access to other people's mental states. That is one reason many people go to counselors: to have their misconceptions about themselves corrected by an authoritative external observer (who in turn relies on the subject's first-person reports, among other sources of evidence). This is also how DES works: the investigator interviews the subject; in the course of the interview, the investigator tries to identify and correct any apparent mistakes made by the subject. In the present context, we are discussing the validity of first-person data-data that result from asking subjects about their mind. Therefore, in the present context, external observers have equal or more accurate access to people's minds. Furthermore, nothing in this objection establishes that first-person data are private in the relevant sense.

The second objection helps us see that to avoid a merely verbal dispute, the term 'private' must be used in the relevant sense. Some of what privatists like to stress is consistent with publicism. For instance, privatists point out that (i) mental states are experienced only by one subject, (ii) subjects have more

\footnotetext{
${ }^{8}$ This objection was inspired by an exchange with Eric Schwitzgebel. Cf.: http://consciousnessonline.wordpress.com/2009/02/20/first-person-data-publicity-and-self-measurement/ ${ }^{9}$ Unless we identify the data with the mental states. That would make first-person data "private" by definitional fiat. But that would still be irrelevant to the sense of privacy relevant to our methodological question. For in the ordinary sense of "data"-l.e., pieces of information about something-data generated through methods that rely on subjective reports are still public. Even mental states are public in the relevant sense, that is, they are observable by public scientific methods, such as DES, albeit less directly than by the subjects themselves.
} 
direct access to their mental states than external observers, and (iii) first-person data are produced by subjects by directly accessing their own mental states. But no combination of these theses amounts to what's at issue here. For these theses are consistent with publicism as defined here, i.e., the view that first-person data are public data and that scientists obtain first-person data by following public methods.

In addition to the above, uncontroversial theses, privatists claim that (i) first-person data cannot be replicated by different observers; therefore, (ii) first-person data cannot be publicly validated; and therefore, (iii) methods that rely on first-person data are "first-person" or private. These theses constitute privatism in the relevant sense.

The temptation to endorse privatism is of a piece with regarding the subjects (as opposed to those who interview them) as the scientific observers, and the subjects' mental states as the data. Given some people's attachment to their actual or purported self-knowledge, this temptation is understandable. The same temptation is reinforced by the methodologies of old-school introspectionist psychologists, phenomenologists, and assorted philosophers, who advocated privatism more or less explicitly. But we should not translate our introspective pride into a piece of scientific methodology. We should recognize that in science, the role of observer is played by the scientists, and scientific data are pieces of information collected by scientists. Once we see this, we see that scientific data-whether first-person or not-are public and, therefore, can be validated by public means. Thus, methods that use firstperson data are as public as other scientific methods.

\section{Why Method Publicity Makes a Difference to Experimental Practice}

As we saw, Schwitzgebel maintains that "[o]ne looks in vain for any genuine prescriptive differences, any study or method permitted by Goldman or Chalmers, forbidden by Dennett (as Dennett himself notes)." It will be useful to separate the two claims: first, that there is no prescriptive difference between privatism and publicism; second, that the same set of studies is permitted by privatism and publicism (for a defense of the second claim, see van de Laar 2008). The second claim is a special case of the first.

Whether a study is permitted is not the most pertinent question. It would be pertinent if there were methods that both parties agree are private. If so, privatists would allow the private methods while publicists would reject them. That would be a prescriptive difference between the two views. But that's not the situation we're in. For we disagree whether reliance on first-person reports and other firstperson data constitute a public or a private method. Still, there are prescriptive differences between the two views.

The questions we should ask are the following. Which view leads to more rigorous, less biased, and more reliable experimental practices? How does each methodology suggest taking precautions against experimental artifacts, searching for and ruling out confounding factors (such as delusion, confabulation, etc.), making explicit assumptions in generating the data, and articulating explicit procedures for encoding the data? Which methodology recommends pursuing research programs aimed at publicly validating (or invalidating, as the case may be) the data? 
Publicism maintains that first-person data are public, like other scientific data. Thus, ceteris paribus, given publicism first-person data should be produced with as much rigor, scrutinized as carefully, and validated as much as other scientific data. The burden of doing so falls on the external observers, who should follow the same strategies they would follow when producing any other data (modulo the peculiarities of the subject matter).

By contrast, privatism maintains that first-person data are private-no one besides the subject has access to them. Because of this, the only case in which first-person data can be invalidated is when a subject contradicts herself (Goldman 1997, 543). Given privatism, strictly speaking, the only people who can take precautions against experimental artifacts and confounding factors are not the external observers but the subjects. Besides noticing inconsistencies, external observers cannot do anything to either validate or invalidate first-person data (Chalmers 2004, Gertler 2009, Goldman 1997, Hatfield 2005).

It is prima facie doubtful that subjects can be relied on to avoid confounding factors and experimental artifacts. For starters, to anyone but the most committed privatist, there is evidence that people's reports about their experience are often faulty. Both Hurlburt and Schwitzgebel explicitly agree on this point.

In addition, the privatist's self-contradiction test for invalid data is faulty. Subjects who give first-person reports, or at least subjects of DES studies, contradict themselves a lot (Hurlburt, personal communication).$^{10}$ It doesn't follow that when they do so they provide no useful information. The correct response to self-contradiction is not to discard the data, but to probe further. One of the contradictory statements is probably more accurate than the other. To determine what happened in the subject's mind-to determine which, if any, of the contradictory statements is more accuratethree things may be done: ask more questions to the subject, appeal to independent evidence, and do more studies of the same kind (e.g., to do more beeping in a DES study). All these actions would be recommended by publicism. They are a clear case of prescriptive differences between privatism and publicism.

How can subjects minimize the risk of experimental artifacts and confounding factors, according to privatists? The most explicit proposal is known as "neurophenomenology" (Varela 1996). Neurophenomenology requires that subjects produce reports about their mind only upon performing a "phenomenological reduction". Roughly speaking, phenomenological reduction is the suspension of our ordinary beliefs about the relation between our experience and the world. But from a publicist point of view, phenomenological reduction is of dubious utility and may even be harmful.

To begin with, it is not clear that (most) people can suspend their beliefs about the relation between their experience and the world. Since such suspension of belief is a private event, given privatism there is no way to check from the outside whether it occurs. So even if phenomenological reduction did indeed minimize experimental artifacts and confounding factors, there would be no way for external observers to collect evidence that such minimization is in place. This is not a promising way to validate

${ }^{10}$ I owe this argument against the self-contradiction test to Russell Hurlburt. 
scientific data. In addition, there is little if any reason to believe that phenomenological reduction minimizes the risk of experimental artifacts and confounding factors. Why should it? If anything, it may be a confounding factor itself. For whatever subjects do upon being told to perform a phenomenological reduction, their efforts may drain attentional and cognitive resources away from the tasks under investigation, thereby interfering with the tasks themselves. Thus, phenomenological reduction appears of dubious utility and possibly harmful.

Objection: you are taking the privatists' methodological statements too literally. When self-avowed "neurophenomenologists" perform actual experiments (e.g., Lutz et al. 2002), phenomenological reduction plays no role in their methods. It's not even mentioned. Instead, it's the experimenters (rather than the subjects) who look for confounders, try to avoid experimental artifacts, and perform statistical tests in line with standard scientific methodology. Experimenters may even look for reliable correlations between first-person data and third-person data, thereby offering independent evidence that the first-person data are valid.

Reply: True, at least in some cases. What this shows is that when methodological push comes to experimental shove, at least some privatists do not behave as privatists. Rather than applying their own methodological prescriptions, they follow standard, third-person methodology. This is all the more reason to reject privatism. But there are two caveats. First, there is no guarantee that all privatists will apply standard methodological checks in their studies-especially when their own methodology does not prescribe them because it deems them impossible. To use Hurlburt's nice phrase, there is still plenty of "armchair introspection" around. Second, methodological rigor comes in degrees. Even if you are a privatist in name only-someone who in fact practices publicist methodology-your background privatism may still incline you towards cavalier reliance on subjective reports, as opposed to checking data as much as you can and striving for public validation. For example, Lutz et al.'s study (2002), which is often cited as a paradigmatic example of neurophenomenology, is especially opaque on how the firstperson data were collected and clustered. The authors do not say how often subjects described their experience one way or another, what assumptions were made in encoding the data, how many experimenters encoded the data, how much the encoders agreed in their clustering of the data, and whether the encoders were blind to the hypothesis being tested. Ideally, publicism would recommend processing the data so as to maximize their publicity-that is, making explicit assumptions about how to cluster the data and having two encoders, blind to the hypothesis being tested, encode the data independently of one another. These procedures would contribute to maximizing data publicity and minimizing sources of bias during the phases of data collection and encoding. As we have seen, whether or not the authors proceeded in the way recommended by publicism, they did not see the need to inform their readers. This may be a consequence of their self-avowed privatism.

In conclusion, privatism and publicism do differ in their prescriptions-and the publicist prescriptions are the ones that maximize the validity of the data.

\section{Descriptive Experience Sampling Is a Public Method}


By now it should be clear why Descriptive Experience Sampling (DES) is a public method. A public method is a method that can be applied by different investigators to answer the same questions, and that when so used, it yields the same results. DES involves a subject and at least one investigator. The investigator asks the subject to describe her experience when a random beeper beeps, then interviews her in more detail about her descriptions. Anyone with the right training can apply DES to answer the same questions, by interviewing the same subject about the same experiences.

Setting aside Schwitzgebel's lack of prior training in DES, the interviews transcribed in Hurlburt and Schwitzgebel's book are an example of method publicity at work. The two of them work together to investigate the contents of Melanie's mind by jointly interviewing her. They also often disagree about the contents of Melanie's mind. Does this show that DES has at least some element of privacy?

Not quite. There is a difference between a method being public and a method being reliable. Hurlburt and Schwitzgebel's disagreement does not show that DES is private. What it shows is that Hurlburt and Schwitzgebel do not take DES to be reliable to the same extent. They make different assumptions about what can be discovered through DES. While Schwitzgebel agrees that DES can be used to uncover some basic features of people's experience, albeit "tentatively" and "pending further evidence" (221), he doubts many of the more detailed conclusions that Hurlburt draws about Melanie's mind. If Schwitzgebel trusted DES as much as Hurlburt and asked the same questions, he would get the same results.

Questioning a method's reliability and the validity of the data obtained through it are important parts of the scientific dialectic. Far from being symptoms of privacy, they lead to further methodological checks - making the method more rigorous - and to data validation studies. In this respect, Schwitzgebel is performing a useful service. By questioning some of Hurlburt's conclusions, he is pushing DES users to make their method more rigorous and uncover independent evidence of data validity.

Looking at the way data are validated (or invalidated) is another way to tell whether a method is public. If the data are validated at best by "plausibility considerations," without any independent (public) evidence, then the method is private. For instance, privatists sometimes argue that "introspection" is legitimate because it's a reliable method, but they don't offer public evidence of reliability-indeed, they can't offer any such evidence, on pain of contradicting their privatism (Goldman 1997, Chalmers 2004, Hatfield 2005). (On the contrary, there is plenty of evidence that introspection is often unreliable, and even if there were no evidence one way or the other, this would hardly be enough to conclude that introspection is reliable.)

By contrast, if data are validated (or invalidated) by appealing to independent (public) evidence, then the method is public. By this standard too, DES is public, and both Hurlburt and Schwitzgebel treat it as such.

To be sure, Hurlburt does present some plausibility arguments in favor of DES, and a few of Hurlburt's considerations are unconvincing. For instance, pointing out that "DES subjects say they give accurate and complete reports" (28) adds little if any credibility to the reports themselves. But the bulk of 
Hurlburt's arguments are different from the typical blanket assumption of reliability made by privatists. Instead, Hurlburt offers (public) evidence that DES is reliable and methodological prescriptions to ensure that DES data are reproducible. Such prescriptions strive to eliminate many potential sources of bias and error by, among other guidelines, asking subjects to describe experience with little delay, targeting specific experiential episodes, keeping the target experience brief, not asking subjects to infer causation, and separating reports from interpretations (14-20). Other arguments include that DES reports are consistent with independently established aspects of experiences, such as attentional limits (29), and that when more than one investigator interviews the same subject, their conclusions agree (33).

While a few of Hurlburt's arguments are unpersuasive, most of them make the beginning of a good case, based on public evidence and the use of public procedures, that DES is a reliable method producing valid data. And these arguments are not even Hurlburt's main source of support for DES: "I do not think that arguments based on plausibility are ever an adequate foundation for science. Science must be based on direct observation, not plausibility" (27). The plausibility arguments merely "set the stage" for "compelling" or "convincing idiographic observations" (27). This leads to two questions: who is doing the "direct" observing? And what makes the idiographic observations "compelling"? Hurlburt's implicit answers are in line with publicism: the psychologists are the direct observers, and what makes the observations compelling is independent, public evidence of their correctness.

Consider the case of Fran, which Hurlburt briefly describes (32ff.). Fran's DES reports led Hurlburt to conclude that, unlike most people, Fran could experience up to ten visual images simultaneously. Even more strikingly, Fran's images appeared to last for hours or days, without interruption. Finally, based on Fran's reports, Hurlburt concluded that Fran had no figure-ground phenomenon in either her imagination or her external perception.

These observations led to a testable prediction. Hurlburt predicted that Fran would not experience the standard alternation between different interpretations of ambiguous figures (such as the vase/faces or the duck/rabbit). Hurlburt tested and confirmed this prediction. Given Fran's idiosyncratic reports, someone might question whether she understood the question about figure vs. ground. Against this worry, Hurlburt points out that Fran was able to describe correctly the difference between her experience and the way other people experience figure vs. ground.

The same DES-based conclusions led to explaining some of Fran's unusual behaviors, which were discovered independently of her DES reports. For instance, Fran used to watch three television sets at once, and she was able to count money, participate in a conversation, and listen to one or more other conversations at the same time. This is (public) behavioral evidence that correlates with, and is explained by, the DES-based conclusion that Fran could sustain several streams of conscious cognitive processing at once.

Finally, Fran's reports about her unusual multiple streams of experience correlated with her borderline personality symptoms, i.e., "exterior disorganization and chaotic psychological fragility" (34). When her symptoms improved, her experiences became "less complex" and she was able to experience figureground phenomena. 
In sum, Hurlburt's DES-based conclusions about Fran's experience correlated with her other behaviors, explained unusual features of her behavior, and were confirmed by a testable prediction. This is remarkable (public) evidence in favor of Hurlburt's DES-based conclusions, which in turn validates the DES data on which the conclusions were based.

As to Schwitzgebel, he explicitly maintains that while validating first-person data publicly is difficult, it is possible. He calls for corroboration of DES data by independent, externally observable evidence $(47,93$, $223,227)$. If Schwitzgebel did not take DES data to be public, he should not be calling for their public validation-for as we saw in Section 2, private data cannot be publicly validated.

Many of Hurlburt and Schwitzgebel's discussions bring in independent, public evidence to either bolster or undermine conclusions derived through DES. For example, they discuss how to show that color experiences are associated with emotions by means of independent evidence (72-3), independent evidence that Melanie's images are more or less detailed $(96-7,102)$, and memory reliability and its role in DES $(149,235-6)$. And in his final reflections, to address some of Schwitzgebel's concerns, Hurlburt suggests studies that could be performed to validate DES data $(275-6,284-5)$. All of this goes to show not only that DES is a public method, but also that both Hurlburt and Schwitzgebel treat it as such.

\section{Conclusion}

Whether a putative scientific method is public is not an idle issue. It has practical repercussions on scientific practices. Unlike privatism, method publicity recommends that investigators (i.e., external observers) take precautions against experimental artifacts, search for and minimize the risk of confounding factors, make explicit their assumptions, articulate explicit procedures for encoding the data, and whenever possible, pursue research programs aimed at publicly validating the data. All of this is part of good science.

Because of this, scientific methods must be public-different investigators must be able to follow the same methods to answer the same questions obtaining the same results. Method publicity is one of the methodological requirements that keep pseudo-science and bad science at bay.

DES is legitimate because it is a public method, yielding public data. While Hurlburt and Schwitzgebel often disagree about which specific conclusions may be legitimately drawn using DES, their discussion is based on independent, public evidence. And the means they identify to resolve their disagreement also rely on public evidence. In spite of their professed neutrality on whether DES is public, commendably, they treat DES as the public method that it is.

\section{References}

Chalmers, D. J. (2004). How Can We Construct a Science of Consciousness? In M. S. Gazzaniga (Ed.), The Cognitive Neurosciences III (pp. 1111-1119). Cambridge, MA: MIT Press.

Dennett, D. C. (2007). Heterophenomenology Reconsidered. Phenomenology and the Cognitive Sciences, 6, 247-270.

Ericsson, K. A. and H. A. Simon (1993). Protocol Analysis: Verbal Reports as Data. Cambridge, MA:MIT

Press. 
Gertler, B. (2009). Introspection. In T. Bayne, A. Cleeremans and P. Wilken (Eds.), The Oxford Companion to Consciousness. Oxford: Oxford University Press.

Goldman, A. (1997). Science, Publicity, and Consciousness. Philosophy of Science, 64, 525-545.

Hatfield, G. (2005). Instrospective Evidence in Psychology. In P. Achinstein (Ed.), Scientific Evidence: Philosophical Theories and Applications (pp. 259-286). Baltimore: Johns Hopkins University Press.

Haybron, D. (2008). The Pursuit of Unhappiness: The Elusive Psychology of Well-Being. Oxford: University of Oxford Press.

Hurlburt, R. and E. Schwitzgebel (2007). Describing Inner Experience: Proponent Meets Skeptic. Cambridge, MA: MIT Press.

Jack, A. I. and A. Roepstorff (2002). Introspection and Cognitive Brain Mapping: From Stimulus-Response to Script-Report. Trends in Cognitive Sciences, 6, 333-339.

Lutz, A., J.-P. Lachaux, J. Martinerie, and F. Varela (2002). Guiding the Study of Brain Dynamics by Using First-Person Data: Synchrony Patterns Correlate With Ongoing Conscious States During a Simple Visual Task. Proceedings of the National Academy of Sciences USA, 99, 1586-1591.

Nahmias, E. A. (2002). Verbal Reports on the Contents of Consciousness: Reconsidering Introspectionist Methodology. Psyche, 8: http://psyche.cs.monash.edu.au/v8/psyche-8-21-nahmias.html.

Piccinini, G. (2003a). Data from Introspective Reports: Upgrading from Commonsense to Science. Journal of Consciousness Studies, 10, 141-156.

Piccinini, G. (2003b). Epistemic Divergence and the Publicity of Scientific Methods. Studies in the History and Philosophy of Science, 34, 597-612.

Piccinini, G. (2009). "First-person Data, Publicity, and Self-Measurement." Philosophers' Imprint 9(9): 116.

van de Laar, T. (2008). Mind the Methodology: Comparing Heterophenomenology and Neurophenomenology as Methodologies for the Scientific Study of Consciousness. Theory and Psychology, 18, 365-379.

Varela, F. (1996). "Neurophenomenology: A Methodological Remedy for the Hard Problem." Journal of Consciousness Studies 3(4): 330-349.

Varela, F. J. and J. Shear (1999). First Person Methodologies: What, Why, How? Journal of Consciousness Studies, 6, 1-14. 